\title{
Validation of the German Version of the Power of Food Scale in a General Population Sample
}

\author{
Elena Andreeva ${ }^{a} \quad$ Maria Neumann ${ }^{b} \quad$ Mariel Nöhre $^{c} \quad$ Elmar Brähler ${ }^{d}$ \\ Anja Hilbert ${ }^{\mathrm{e}}$ Martina de Zwaan ${ }^{c}$ \\ ${ }^{a}$ Centre for Applied Rehabilitation Research, Department of Rehabilitation Medicine, \\ Hannover Medical School, Hannover, Germany; ${ }^{b}$ Equal Opportunities Office, Hannover \\ Medical School, Hannover, Germany; ${ }^{\mathrm{C}}$ Department of Psychosomatic Medicine and \\ Psychotherapy, Hannover Medical School, Hannover, Germany; ${ }^{\mathrm{d} D e p a r t m e n t}$ of \\ Psychosomatic Medicine and Psychotherapy, Johannes Gutenberg University, Mainz,

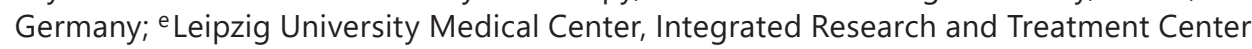 \\ AdiposityDiseases, Medical Psychology and Medical Sociology, Psychosomatic Medicine \\ and Psychotherapy, Leipzig, Germany
}

\section{Keywords}

Power of Food Scale · Validation · German version

\begin{abstract}
Objective: The Power of Food Scale (PFS) is a self-report instrument for assessing appetitive motivation in the absence of caloric needs. The study aim was to validate the German PFS version in a large population sample. Methods: Complete information on all PFS items was available from 2,421 respondents (age $\geq 14$ ) of a nationally representative sample of the German population. We examined the psychometric properties of the German PFS version and provided population-based normative data. Results: The 3-factor structure of the original scale was replicated in confirmatory factor analysis. The German PFS version demonstrated good internal consistency ( $\alpha=0.92$ for the total scale). It was well accepted by the respondents, as indicated by a low proportion of missing item values $(\leq 0.56 \%)$. While no significant differences were observed in the PFS mean scores between men and women, the scores increased across BMI categories. PFS was positively correlated with a measure of global eating disorder psychopathology (Eating Disorder Examination-Questionnaire 8) and the ultra-brief Patient Health Questionnaire for depression and anxiety. Conclusions: Our findings suggest that the German PFS version has adequate psychometric properties and good reliability for measuring hedonic hunger in the general population. The provided population-based norms can be used for individual assessment.


Andreeva et al.: German Version of the Power of Food Scale

\section{Introduction}

Overweight and obesity are risk factors for various health problems including diabetes mellitus, cardiovascular diseases, and cancer. According to recent estimates, $67.1 \%$ of men and $53.0 \%$ women in Germany suffer from obesity or overweight [1]. Obesity and overweight result from a chronic positive balance between food intake and energy expenditure. Eating behavior is regulated by homeostatic needs, emotional cues, dietary restraints, social and environmental factors, but also by the experience of pleasure associated with food consumption $[2,3]$. The term "hedonic hunger" was coined to refer to one's desire to consume food for pleasure in the absence of caloric need [4]. Elevated hedonic hunger is common among patients with severe obesity [5]. It may also predict the development or maintenance of binge eating, defined as consuming large amounts of food within a discrete time period while experiencing a sense of loss of control (LOC) over eating [6, 7].

For the assessment of individual variations in hedonic hunger, the self-report measure Power of Food Scale (PFS) was developed addressing the appetitive motivation in response to food-abundant environments, as reflected by feelings of being controlled by food independent of actual caloric intake [8]. Accordingly, the PFS is assumed to identify risk groups for overeating in the modern obesogenic environment, in which the ubiquity of palatable energydense foods provokes the experience of hedonic hunger $[8,9]$. This assumption is based on a two-factor model of appetite suggesting that individual differences in motivation to eat beyond caloric need will be exaggerated in environments, in which the proximity of food to people affects their appetitive reactions [4, 10,11]. Lowe and Butryn [4] distinguished between the homeostatic and hedonic motives of food intake. According to the authors, the homeostatic pathway regulates energy balance by increasing the motivation to eat when readily available energy sources are running low. In contrast, hedonic or reward-based regulation increases the desire to eat highly palatable food in order to obtain pleasure also in periods of relative energy abundance. Although both drives are complementary, there is evidence that individuals with obesity prefer highly palatable foods more than persons of normal weight. The PFS domains were designed to measure the appetitive impact of palatable food at three levels of proximity. The first level refers to the widespread availability of palatable food in the environment: food is available but not physically present. The second level implies to appetitive reactions to food physically present but not yet tasted. The third level involves reactions to food when it is already tasted but not yet consumed [8]. Previous research identified a three-factor structure of the PFS, with the factors corresponding to the domains above $[12,13]$. By now, the PFS has been widely used, validated and translated into many languages including Chinese, Japanese, and Portuguese [13-15]. To our knowledge, no validated German version of the PFS is available so far. Hence, the primary purpose of the present study was to validate the German version of the PFS after translation of the original English questionnaire [8]. We aimed to replicate the three main PFS domains in a representative sample of the German population [12].

Based on earlier studies, the PFS appears to be sensitive to detecting eating disorder psychopathology that may increase the risk of adverse health outcomes at all levels of body mass $[2,6]$. Since eating disorders often co-occur with symptoms of anxiety and depression $[16,17]$, we hypothesized positive relationships between the PFS scores, eating-related psychopathology as well as anxiety and depression. To our knowledge, the associations of the PFS with anxiety and depression have not been explicitly studied. Therefore, our study sought to (a) examine the distribution of the PFS scores across age groups, sexes and BMI (body mass index) ranges, (b) confirm the positive relationships between the PFS scores and eatingrelated psychopathology, and (c) explore the association between the PFS and anxiety and depression. Finally, we aimed at providing the population-based norms for the German general population, as it has been reported before for the Portuguese version of the PFS [13]. 
Andreeva et al.: German Version of the Power of Food Scale

\section{Materials and Methods}

\section{Study Sample}

A random sample of German residents aged 14 years and older was selected for a crosssectional survey on physical and mental population well-being. A demographic consulting company USUMA (Berlin) assisted with the sampling and data collection. According to the established procedure on data collection without any access to population registers, the sampling design included three consecutive steps. First, a sample of 258 living areas was randomly selected from a nonoverlapping stratum of all area units: 210 areas were sampled from Western Germany and 48 areas from Eastern Germany. The random selection of households was implemented in the second step. Finally, one person matching the inclusion criteria (age $\geq 14$ and fluent in German) was randomly selected from each household. The procedure was designed to yield the national sample representative in terms of age, gender, and education over the fieldwork period from September through November 2016. Sociodemographic data were collected by trained interviewers face-to-face. In addition, participants returned a battery of self-report questionnaires including the German PFS version. Of the preselected 4,902 addresses, 4,838 were valid. The participation rate of $52 \%$ takes into account all refusals by respondents, as well as noninterviews due to respondents' illness or unavailability during the fieldwork. All in all, 2,514 persons returned interviews. Because of completely missing PFS data, we excluded 13 interviews. Thus, the final sample consisted of 2,501 persons.

\section{Measures}

The agreement to use the PFS was obtained from Drexel University. The original PFS [8] was translated into German language by Anja Hilbert [unpubl. manuscript] in discussion with Martina de Zwaan and in accordance with the recommended procedure [18] including an adaptation to the sociocultural context of the target language. Furthermore, back-translation was performed by an independent bilingual licensed translator in order to uncover the potential ambiguity or misunderstanding in the first translation. The German PFS version includes 15 items for measuring hedonic hunger as a stable trait. Each item is scored on a fivepoint Likert scale from $1=$ do not agree at all to $5=$ strongly agree, with higher scores indicating greater responsiveness to living in food-abundant environments [4]. In agreement with earlier research $[8,12]$, subscales for each domain were obtained as a mean score of all items included in the subscale (range $=1-5$ ). Similarly, the aggregate score was calculated as an average score for all 15 items (range $=1-5$ ). The internal consistency of the original PFS was very good with a Cronbach's $\alpha$ of 0.91 [8], considering 0.70 as an acceptable and 0.90 as a recommended level for assessment scales [19].

The ultra-brief 4-item Patient Health Questionnaire for Depression and Anxiety (PHQ-4) was used to assess the core symptoms of depression and anxiety disorder [20,21]. Respondents rated how often, on a scale from $0=$ not at all to $3=$ nearly every day, they experienced feeling depressed, loss of interest, feeling nervous or anxious, and inability to stop worrying over the past 2 weeks. Therefore, the total score ranges from 0 to 12. The PHQ-4 has shown a good internal reliability with Cronbach's $\alpha>0.80$ for all scales [21]. In our sample, Cronbach's alpha was $\alpha=0.88$.

The Eating Disorder Examination-Questionnaire 8 (EDE-Q8) provides a condensed assessment of the global eating disorder psychopathology [22]. The questionnaire includes eight items asking respondents how often - on a scale from $0=$ not at all to $6=$ every day or in extreme form - they experienced restraint over eating, food avoidance, preoccupation with food, feeling of being fat, desire to lose weight, guilt about eating, dissatisfaction with weight, and discomfort seeing their body during the last 4 weeks. The global mean score ranges from 
0 to 6. The EDE-Q8 demonstrated good item characteristics and reliability, with Cronbach's $\alpha=0.93$ [22]. In our sample, the internal reliability was comparably high $(\alpha=0.91)$.

Data on body weight and height were provided as self-report, the BMI was calculated using the formula: BMI = body weight $(\mathrm{kg}) /\left(\right.$ body height $\left.(\mathrm{m})^{2}\right)$. The BMI was categorized into underweight/normal weight $(\mathrm{BMI}<25)$, overweight $(25 \leq \mathrm{BMI}<30)$, and obesity $(\mathrm{BMI} \geq 30)$ [23].

\section{Statistical Analysis}

Statistical analyses were performed using STATA MP 14.1 and SPSS 24. Significance was considered at $p<0.05$. First, we examined the item descriptive statistics, percentage of missing values, corrected item-total correlations, and item difficulties (in \%) using the formula: $\mathrm{p}_{\mathrm{i}}=\left(\left(\operatorname{mean}\left(\mathrm{x}_{\mathrm{i}}\right)-\min \left(\mathrm{x}_{\mathrm{i}}\right)\right) /\left(\max \left(\mathrm{x}_{\mathrm{i}}\right)-\min \left(\mathrm{x}_{\mathrm{i}}\right)\right) \times 100\right.$, with mean $\left(\mathrm{x}_{\mathrm{i}}\right)=$ mean of item $\mathrm{i} ; \min \left(\mathrm{x}_{\mathrm{i}}\right)=$ minimal value of item $\mathrm{i} ; \max \left(\mathrm{x}_{\mathrm{i}}\right)=$ maximal value of item $\mathrm{i}$. In the second step, we examined the internal consistency of the subscales and the total scale using Cronbach's $\alpha$.

Confirmatory factor analysis was performed to verify that the factor structure of the German PFS is the same as that in the original PFS. The analysis was conducted using the maximum likelihood estimation algorithm with robust standard errors (option Structural Equation Modelling in STATA). As in earlier studies, we used a three-factor model with the latent variables Food available (items 1, 2, 5, 10, 11, and 13), Food present (items 3, 4, 6, and 7) and Food tasted (items 8, 9, 12, 14, and 15) denoting the three levels of proximity of palatable food in the environment $[12,15]$. Each item was specified to load on a single factor, as suggested in prior research [12]. Model fit was assessed by multiple criteria. Absolute fit was determined by the $\chi^{2}$, the standardized root mean square residual (SRMR) and the root mean square of approximation (RMSEA) including the $90 \%$ confidence interval. The Comparative Fit Index (CFI) and the Tucker-Lewis Index (TLI) were used as comparative fit indices. A good model fit corresponds to CFI and TLI values $>0.95$ ( 0.90 is acceptable), SRMR $<0.05$ ( 0.09 is acceptable), and RMSEA values $<0.05$ (values between 0.05 and 0.08 are acceptable) [24].

Means and standard deviations (SD) were calculated for the total PFS and its subscales in the total sample and across gender, age, and BMI categories. The differences across categories were analyzed by means of nonparametric Kruskal-Wallis test.

The Spearman's rank correlation coefficients were calculated to examine the relationships between the PFS scores, eating disorder psychopathology (EDE-Q8), and anxiety and depression (PHQ-4). Finally, in order to report normative data, we calculated percentile ranks based on the total mean score of the PFS and its subscales by gender, age, and BMI categories.

\section{Results}

\section{Sample Description}

Table 1 shows the sociodemographic characteristics of our respondents. The analytic sample included almost equal proportions of men $(46.7 \% ; n=1,167)$ and women $(53.3 \%$; $n=1,334$ ) aged between 14 and 94 years (mean = 48.4; SD = 18.2). Mean BMI was 25.7 (SD = 4.6); approximately half of the respondents had overweight and obesity $(50.6 \% ; n=1,244)$. The majority of the sample reported residence in Western Germany $(79.9 \% ; n=1,998)$, living in a partnership (55.0\%; $n=1,364)$, and having an income of EUR $\geq 1,200(83.4 \% ; n=1,998)$. In terms of educational level, more than one fifth of the sample stated the overall duration of $\geq 12$ years of school attendance $(21.7 \% ; n=541)$. A minority were unemployed $(5.2 \% ; n=$ $130)$ and reported a non-German nationality $(3.2 \% ; n=81)$. 
Andreeva et al.: German Version of the Power of Food Scale

Table 1. Sociodemographic characteristics of the sample

\begin{tabular}{|c|c|c|c|}
\hline & $\begin{array}{l}\text { Total } \\
(n=2,501)\end{array}$ & $\begin{array}{l}\text { Women } \\
(n=1,334)\end{array}$ & $\begin{array}{l}\text { Men } \\
(n=1,167)\end{array}$ \\
\hline \multicolumn{4}{|l|}{ Age, years } \\
\hline$\leq 24$ & $280(11.2)$ & $134(10.0)$ & $146(12.5)$ \\
\hline $25-34$ & $390(15.6)$ & $202(15.1)$ & $188(16.1)$ \\
\hline $35-44$ & $385(15.4)$ & $221(16.6)$ & $164(14.0)$ \\
\hline $45-54$ & $464(18.5)$ & $249(18.7)$ & $215(18.4)$ \\
\hline $55-64$ & $434(17.3)$ & $229(17.2)$ & 205 (17.6) \\
\hline $65-74$ & 327 (13.1) & 177 (13.3) & $150(12.8)$ \\
\hline$\geq 75$ & $221(8.8)$ & $122(9.1)$ & $99(8.5)$ \\
\hline \multicolumn{4}{|l|}{ Residence } \\
\hline Eastern Germany & $503(20.1)$ & $254(19.0)$ & 249 (21.3) \\
\hline Western Germany & $1,998(79.9)$ & $1,080(81.0)$ & 918 (78.7) \\
\hline \multicolumn{4}{|l|}{ Settlement type } \\
\hline Urban & $2,182(87.2)$ & $1,172(87.9)$ & $1,010(86.5)$ \\
\hline Rural & 319 (12.8) & $162(12.1)$ & $157(13.5)$ \\
\hline \multicolumn{4}{|l|}{ Nationality } \\
\hline German & $2,420(96.8)$ & $1,298(97.3)$ & $1,122(96.1)$ \\
\hline Other & $81(3.2)$ & $36(2.7)$ & $45(3.9)$ \\
\hline \multicolumn{4}{|l|}{ Educational level } \\
\hline$<12$ years & $1,950(78.3)$ & 1,057 (79.7) & 893 (76.7) \\
\hline$\geq 12$ years & $541(21.7)$ & $270(20.3)$ & $271(23.3)$ \\
\hline \multicolumn{4}{|l|}{ Employment status } \\
\hline Not unemployed & $2,352(94.8)$ & $1,261(95.2)$ & $1,091(94.2)$ \\
\hline Unemployed & $130(5.2)$ & $63(4.8)$ & $67(5.8)$ \\
\hline \multicolumn{4}{|l|}{ Household income } \\
\hline$<1,250$ EUR & $398(16.6)$ & $241(18.8)$ & $157(14.1)$ \\
\hline 1,250-2,500 EUR & $968(40.4)$ & $532(41.5)$ & $436(39.1)$ \\
\hline$>2,500$ EUR & $1,030(43.0)$ & 509 (39.7) & $521(46.8)$ \\
\hline \multicolumn{4}{|l|}{ Living with a partner } \\
\hline Yes & $1,364(55.0)$ & $716(54.0)$ & $648(56.2)$ \\
\hline No & $1,116(45.0)$ & $610(46.0)$ & $506(43.8)$ \\
\hline \multicolumn{4}{|l|}{ Body mass index } \\
\hline Underweight/normal weight & $1,215(49.4)$ & $722(55.2)$ & $493(42.8)$ \\
\hline Overweight & $905(36.8)$ & 391 (29.9) & $514(44.7)$ \\
\hline Obesity & $339(13.8)$ & 195 (14.9) & $144(12.5)$ \\
\hline
\end{tabular}

\section{Characteristics of Items and Subscales}

Complete information on all items of the PFS was available from 2,421 respondents. The item characteristics are displayed in Table 2 . The percentage of missing values was very low, with a maximum of $0.6 \%$ (item 11 ) and a mean percentage of $0.4 \%$ (SD $=0.11$ ) for all items. Almost $41.0 \%$ (SD $=12.6)$ of the score values per subscale fell into the lowest category (do not agree at all). The subscale (domain) Food available demonstrated the largest proportion of scores in the lowest category (mean $=50.8 \%$; SD $=14.5$ ), whereas the lowest proportion was found for the domain Food tasted (mean $=32.1 \%$; $S D=14$ ). All but one item were positively skewed; the only exception refers to item 14 within the domain Food tasted. The kurtosis was positive for all items. Item difficulties ranged between 12.3 and $52.7 \%$, indi- 
Andreeva et al.: German Version of the Power of Food Scale

Table 2. Item and scale characteristics of the German version of the Power of Food Scale

\begin{tabular}{llllllllll}
\hline PFS & Item & Mean & SD & Skewness & Kurtosis & $\mathrm{p}_{\mathrm{i},} \%$ & $\mathrm{r}_{\mathrm{it}}{ }^{\mathrm{a}}$ & \% missing & CFA $^{\mathrm{a}}$ \\
\hline \multirow{2}{*}{ Available } & Q1 & 1.72 & 0.97 & 1.27 & 3.82 & 18.0 & 0.59 & 0.08 & 0.68 \\
& Q2 & 1.82 & 1.00 & 0.98 & 2.98 & 20.4 & 0.59 & 0.36 & 0.65 \\
& Q5 & 1.49 & 0.90 & 1.92 & 6.00 & 12.3 & 0.59 & 0.32 & 0.71 \\
& Q10 & 1.79 & 1.02 & 1.12 & 3.31 & 19.9 & 0.66 & 0.32 & 0.72 \\
& Q11 & 1.93 & 1.10 & 0.94 & 2.85 & 23.2 & 0.59 & 0.56 & 0.62 \\
& Q13 & 1.63 & 0.97 & 1.56 & 4.72 & 15.6 & 0.71 & 0.32 & 0.82 \\
\hline \multirow{2}{*}{ Present } & Q3 & 2.37 & 1.09 & 0.37 & 2.28 & 34.2 & 0.70 & 0.48 & 0.74 \\
& Q4 & 1.98 & 1.11 & 0.89 & 2.81 & 24.5 & 0.68 & 0.32 & 0.77 \\
& Q6 & 1.93 & 1.04 & 0.89 & 2.87 & 23.2 & 0.70 & 0.28 & 0.80 \\
& Q7 & 2.14 & 1.17 & 0.71 & 2.43 & 28.4 & 0.68 & 0.32 & 0.77 \\
\hline \multirow{2}{*}{ Tasted } & Q8 & 2.46 & 1.20 & 0.33 & 2.02 & 36.5 & 0.67 & 0.48 & 0.78 \\
& Q9 & 2.64 & 1.21 & 0.11 & 1.93 & 41.1 & 0.62 & 0.36 & 0.73 \\
& Q12 & 1.96 & 1.07 & 0.84 & 2.75 & 24.0 & 0.69 & 0.40 & 0.70 \\
& Q14 & 3.11 & 1.30 & -0.25 & 1.93 & 52.7 & 0.48 & 0.40 & 0.57 \\
& Q15 & 1.99 & 1.17 & 0.92 & 2.76 & 24.7 & 0.52 & 0.48 & 0.57 \\
\hline
\end{tabular}

PFS, Power of Food Scale; available, subscale Food available; present, subscale Food present; tasted, subscale Food tasted; SD, standard deviation; $p_{i}$, item difficulty; $r_{i t}$ corrected item-total correlation; CFA, confirmatory factor analysis: factor loadings (standardized coefficients); Q1-Q15, item numbering in the German PFS version. ${ }^{a}$ Analyses were conducted for cases with complete PFS data $(n=2,421)$.

cating a low to medium probability of scores $>1$ (do not agree at all). Corrected item-total correlations were moderate-to-high $\left(0.48 \leq \mathrm{r}_{\text {it }} \leq 0.71\right)$.

\section{Factor Structure}

The confirmatory factor analysis showed the following fit indices for the 3-factor solution: $\chi^{2}=1,749.5 ; \mathrm{df}=87 ; \mathrm{CFI}=0.91 ; \mathrm{TLI}=0.89 ; \mathrm{SRMR}=0.05 ;$ RMSEA $=0.089$ (90\% confidence interval: $0.085 ; 0.092)$. The factor loadings are displayed in Table 2 . All of the standardized coefficients were statistically significant $(p<0.001)$ and exceeded 0.4 . The subscale Food available correlated with Food present $(r=0.83)$ and Food tasted $(r=0.76)$. The correlation between Food present and Food tasted was $r=0.83$.

\section{Reliability}

For the total PFS, the mean inter-item correlation was 0.44 with a Cronbach's $\alpha$ of 0.92 . Internal consistency for the subscales turned out to be satisfactory (online suppl. Appendix: Table A1; for all online suppl. material, see www.karger.com/doi/10.1159/000500489: $\alpha=0.85$ for Food available and Food present; $\alpha=0.80$ for Food tasted). For the subscales, the average inter-item correlations were 0.49 for Food available, 0.59 for Food present, and 0.45 for Food tasted, being within the recommended range for tapping the narrower characteristics [25].

\section{Differences in PFS Scores across Gender, Age, and BMI Status}

Table 3 shows the means and SDs for the total PFS and its subscales across gender, age, BMI categories and in the total sample. Overall, the highest mean scores were observed for the subscale Food tasted, followed by Food present and Food available, both within the total sample and for each separate subgroup. We found no significant differences by gender. Further results indicated a decrease in the mean scores by age in respondents above 45-54 years, with significant differences for Food present, Food tasted, and the total score, all $p<$ 
Andreeva et al.: German Version of the Power of Food Scale

Table 3. Mean and standard deviation for the total PFS score and the domain subscores: group comparisons

\begin{tabular}{lllll}
\hline & Available & Present & Tasted & Total mean score \\
\hline Total sample $(n=2,421)$ & $1.73(0.75)$ & $2.11(0.92)$ & $2.44(0.89)$ & $2.07(0.75)$ \\
\hline Sex & $p=0.725$ & $p=0.300$ & $p=0.675$ & $p=0.533$ \\
Men $(n=1,131)$ & $1.72(0.75)$ & $2.09(0.93)$ & $2.43(0.90)$ & $2.06(0.76)$ \\
Women $(n=1,290)$ & $1.73(0.75)$ & $2.12(0.91)$ & $2.44(0.88)$ & $2.07(0.74)$ \\
\hline Age groups & $p=0.074$ & $p<0.001$ & $p<0.001$ & $p<0.001$ \\
$\quad$ 24 $(n=271)$ & $1.76(0.73)$ & $2.17(0.94)$ & $2.43(0.89)$ & $2.09(0.75)$ \\
$\quad$ 25-34 $(n=381)$ & $1.75(0.76)$ & $2.20(0.93)$ & $2.54(0.93)$ & $2.13(0.76)$ \\
35-44 $(n=378)$ & $1.76(0.80)$ & $2.20(0.96)$ & $2.47(0.93)$ & $2.11(0.77)$ \\
$\quad 45-54(n=446)$ & $1.76(0.75)$ & $2.15(0.91)$ & $2.50(0.87)$ & $2.11(0.73)$ \\
$\quad 55-64(n=420)$ & $1.75(0.77)$ & $2.05(0.90)$ & $2.43(0.86)$ & $2.06(0.75)$ \\
$\quad 65-74(n=313)$ & $1.64(0.69)$ & $2.00(0.91)$ & $2.34(0.86)$ & $1.97(0.72)$ \\
$\geq 75(n=212)$ & $1.61(0.67)$ & $1.87(0.79)$ & $2.22(0.85)$ & $1.88(0.67)$ \\
\hline BMI $\quad p<0.001$ & $p<0.001$ & $p<0.001$ & $p<0.001$ \\
$\quad$ Normal weight and underweight & & & \\
$\quad(n=1,180)$ & $1.61(0.65)$ & $1.95(0.83)$ & $2.35(0.88)$ & $1.94(0.68)$ \\
$\quad$ Overweight $(n=871)$ & $1.76(0.77)$ & $2.16(0.93)$ & $2.48(0.90)$ & $2.11(0.76)$ \\
$\quad$ Obesity $(n=331)$ & $2.09(0.87)$ & $2.54(1.01)$ & $2.65(0.85)$ & $2.40(0.82)$ \\
\hline
\end{tabular}

These analyses are restricted to respondents with complete data on the PFS items, age, sex, and BMI. $n$, number of participants with complete responses; available, subscale Food available; present, subscale Food present; tasted, subscale Food tasted. $p$ values for Kruskal-Wallis equality-of-populations rank test (group differences are considered statistically significant if $p<0.05$ ).

0.001. Finally, the scores consistently increased with higher BMI categories, with the largest mean values in respondents with obesity and the lowest scores in those with normal weight and underweight (all $p<0.001$ ). We computed Cohen's $d$ effect size for comparing the differences between the PFS score means across BMI categories (online suppl. Appendix: Table A2). The results indicate medium-size score differences between the categories obesity versus normal weight or underweight for the subscales Food available and Food present as well as for the total score (Cohen's $d$ 0.63-0.68).

\section{Associations of PFS with Measures of Eating Disorder (EDE-Q8), Depression, and Anxiety (PHQ-4)}

Bivariate correlations are displayed in Table 4 for the total sample and by BMI categories. Significant but weak positive associations were found between PFS and PHQ-4; these relationships were strongest in participants with obesity and least strong in persons with normal weight and underweight. The total score of the EDE-Q8 correlated weakly but significantly with the PFS, with little variation across BMI categories, all $p<0.001$.

\section{Normative Data for PFS}

In the total sample, the total mean score was $2.07(\mathrm{SD}=0.75)$, median $=1.93$. The respective figures for the subscore for Food available were: mean $=1.73(\mathrm{SD}=0.75)$, median $=1.5$. The subscore data for Food present were: mean $=2.11(\mathrm{SD}=0.92)$, median $=2$. The average subscore for Food tasted was mean $=2.44(\mathrm{SD}=0.89)$, median $=2.4$. We calculated percentile ranks for the total score and the PFS subscales by gender and age groups (online suppl. Appendix: Tables A3-A4). Finally, given the significant differences in the total score and subscale values by BMI category, we also reported percentile ranks for BMI categories (online suppl. Appendix: Table A5). 
Andreeva et al.: German Version of the Power of Food Scale

Table 4. Two-tailed Spearman's rank correlations between the PFS and the ultra-brief patient questionnaire for depression and anxiety (PHQ-4) as well as the eating disorder examination questionnaire 8 (EDE-Q8)

\begin{tabular}{lllll}
\hline & Total sample & By BMI categories & & \\
\cline { 3 - 5 } & & $\begin{array}{l}\text { normal weight and } \\
\text { underweight }\end{array}$ & overweight & obesity \\
\hline PHQ-4 $(n=2,407)$ & $0.244(p<0.001)$ & $0.209(p<0.001)$ & $0.234(p<0.001)$ & $0.290(p<0.001)$ \\
EDE-Q8 $(n=2,369)$ & $0.326(p<0.001)$ & $0.267(p<0.001)$ & $0.331(p<0.001)$ & $0.287(p<0.001)$
\end{tabular}

Casewise deletion of missing data was used.

\section{Discussion}

The aim of the present study was to validate the German version of the PFS and to provide normative data based on a large representative sample of the German population. Our findings suggest that the PFS was well accepted by the respondents as reflected by the very low proportion of missing values on the items of the questionnaire $(\leq 0.6 \%)$ : thus, items were not difficult to understand. A positively skewed distribution for the majority of items and medium-to-high item difficulties indicates that the PFS focuses on higher levels of hedonic hunger in the absence of caloric needs. There was some evidence of floor effects, as indicated by a high, $>40 \%$ on average, proportion of participants scoring at the lowest response category (do not agree at all). Results for corrected item-total correlations were in agreement with findings for the original PFS: all items were overall well correlated with the scale [8]. Cronbach's $\alpha$ (0.92) for the total scale of the German version was almost identical to that of the English original [8]. For all items and subscales, Cronbach's $\alpha$ exceeded 0.7 , suggesting a very good internal consistency of the German PFS [19, 26].

The construct validity of the German PFS version was examined using confirmatory factor analysis. The model supported a three-factor solution (with the factors Food available, Food present, and Food tasted), showed acceptable CFI and SRMR, significant item loads onto the factors, and high correlations among factors. These results are well in line with prior findings based on the English original $[8,12]$. The indices TLI and RMSEA scored slightly out of the acceptable range; RMSEA was, however, identical to that in previous research [15] and still within the range of mediocre fit, as indicated by the upper limit of the $90 \%$ confidence interval $<0.1$ on the point estimate [27].

The appetitive impact of palatable food appears to grow by levels of proximity, from Food available over Food present to Food tasted, as indicated by the mean PFS scores in all subgroups of our sample. In terms of distributional analysis, we found no notable variance in PFS scores across gender, in accordance with the results described for the English original [8]. Yet, gender differences are discussed, given the higher scores in women from other national samples [15]. The mixed evidence might be attributed to culture-specific gender attitudes to perception of food cues or to differences in sample characteristics. In the latter respect, our research has the advantage of a representative population sample covering nearly the entire life span for both sexes (14-94 years). Our findings of lower mean scores in older age groups compared to younger age groups corroborate earlier research [13]. Most prominent differences by age were found for the subscales Food present and Food tasted. This observation can be interpreted in line with the theory of externality suggesting that some people - in our sample, those in younger age groups - may be more sensitive to external 
food cues and more likely to perceive hedonic hunger in response to sight, smell or taste of food $[28,29]$. Furthermore, our data demonstrate increasing PFS scores across BMI categories. We can therefore assume higher appetitive motivation in individuals with overweight and obesity. Previous research has, however, yielded inconsistent evidence on relations between PFS and measures of excess weight, varying between no [15, 30], weak [8, 31], and strong associations [31]. Importantly, strong associations have emerged before with obesity status, including severe obesity in candidates for bariatric surgery, but not with BMI as a continuous variable [31]. These discrepancies may suggest that the relationships between hedonic hunger and measures of excess weight are mediated by underlying obesity-related behavioral patterns, for example LOC eating. Studies with prospective design are warranted to clarify the role of hedonic hunger in development of LOC eating as a predictor of overweight or obesity.

Findings from the present study contribute to the understanding of PFS as a possible correlate of global eating disorder psychopathology, as measured by the EDE-Q8. The association between hedonic hunger and measures of problematic eating behavior, including disinhibited eating, emotional eating, and food addiction, have also been confirmed earlier [6, 8]. Vainik et al. [32] showed that the PFS reflects the middle on a continuum of an underlying trait termed "uncontrolled eating," with no overeating as one extreme and food addiction and binge eating on the other side. In our sample, significant but weak positive associations imply that, although hedonic hunger and problematic eating reflect different concepts, they may be explained by a shared underlying trait, most likely susceptibility to LOC eating [2], at least in some individuals.

Finally, we observed consistent, albeit weak, positive relationships between the scores of the PFS and the PHQ-4 at all levels of BMI. This finding indicates that hedonic hunger may be coupled with adverse emotional states. This is not surprising, given that appetitive motivation may manifest itself not only as cognitive preoccupation with palatable food, but also in terms of overeating or binge eating in response to emotional distress [16].

A major strength of our study is the use of a large population-based sample representative of the German general population in terms of age and gender. Our research fills a gap in the literature by examining the factor structure of the PFS with a confirmatory approach: to our knowledge, this has not been done before. Furthermore, the provided normative data for the PFS scores should increase the applicability of the German version for use in research and clinical practice. There are, however, several important limitations. First, the crosssectional design precludes assessments of test-retest reliability and causal inferences, e.g. on associations of hedonic hunger with adverse emotional states. Second, concerning criterion validity, we did not compare PFS with any other measures of hedonic hunger in the absence of thereof. Third, due to the self-report nature of the data, the results may be susceptible to common method and social desirability biases. Although we believe the risk was reduced by relying on anonymity and assuring voluntary participation, it is impossible to completely rule out the problem. Thus, the prevalence of overweight and obesity in our study was lower than in the large representative "German Health Interview and Examination Survey for Adults" conducted between 2008 and 2011 with objectively measured body weight and height [1], probably due to underreporting. This discrepancy limits the generalizability of our results.

In summary, our study demonstrates adequate psychometric properties of the German PFS version and very good reliability for measuring hedonic hunger in a large populationbased sample. The provided population-based norms can be used for individual assessment. Prospective studies with BMI based on objective measurement are needed to examine hedonic hunger as a predictor of overweight and obesity. 
Andreeva et al.: German Version of the Power of Food Scale

\section{Acknowledgement}

We thank all participants for study participation.

\section{Statement of Ethics}

All participants provided their written informed consent in accordance with the Helsinki Declaration. For underage persons, the informed consent was additionally given by one of the parents. The study was approved by the Ethics Committee of the University of Leipzig on September 13, 2016.

\section{Disclosure Statement}

The authors have no conflicts of interest to declare.

\section{Funding Sources}

No funding sources.

\section{Author Contributions}

M.Z. and A.H. designed the study. E.A. and M.N. carried out the analyses and prepared the first draft (shared first authorship); E.A. finalized the manuscript. All authors were involved in writing the paper and had final approval of the submitted and published versions.

\section{References}

1 Mensink GB, Schienkiewitz A, Haftenberger M, Lampert T, Ziese T, Scheidt-Nave C. [Overweight and obesity in Germany: results of the German Health Interview and Examination Survey for Adults (DEGS1)]. Bundesgesundheitsblatt Gesundheitsforschung Gesundheitsschutz. 2013 May;56(5-6):786-94.

2 Espel-Huynh HM, Muratore AF, Lowe MR. A narrative review of the construct of hedonic hunger and its measurement by the Power of Food Scale. Obes Sci Pract. 2018 Feb;4(3):238-49.

3 Neumann M, Holzapfel C, Müller A, Hilbert A, Crosby RD, de Zwaan M. Features and Trajectories of Eating Behavior in Weight-Loss Maintenance: Results from the German Weight Control Registry. Obesity (Silver Spring). 2018 Sep;26(9):1501-8.

4 Lowe MR, Butryn ML. Hedonic hunger: a new dimension of appetite? Physiol Behav. 2007 Jul;91(4):432-9.

5 Schultes B, Ernst B, Wilms B, Thurnheer M, Hallschmid M. Hedonic hunger is increased in severely obese patients and is reduced after gastric bypass surgery. Am J Clin Nutr. 2010 Aug;92(2):277-83.

6 Lowe MR, Arigo D, Butryn ML, Gilbert JR, Sarwer D, Stice E. Hedonic hunger prospectively predicts onset and maintenance of loss of control eating among college women. Health Psychol. 2016 Mar;35(3):238-44.

7 American Psychiatric Association, editor. Diagnostic and Statistical Manual of Mental Disorders. 5th ed. Washington (DC): American Psychiatric Association; 2013.

8 Lowe MR, Butryn ML, Didie ER, Annunziato RA, Thomas JG, Crerand CE, et al. The Power of Food Scale. A new measure of the psychological influence of the food environment. Appetite. 2009 Aug;53(1):114-8.

9 Winter SR, Yokum S, Stice E, Osipowicz K, Lowe MR. Elevated reward response to receipt of palatable food predicts future weight variability in healthy-weight adolescents. Am J Clin Nutr. 2017 Apr;105(4):781-9.

10 Lowe MR, Levine AS. Eating motives and the controversy over dieting: eating less than needed versus less than wanted. Obes Res. 2005 May;13(5):797-806.

11 Painter JE, Wansink B, Hieggelke JB. How visibility and convenience influence candy consumption. Appetite. 2002 Jun;38(3):237-8. 
Andreeva et al.: German Version of the Power of Food Scale

12 Cappelleri JC, Bushmakin AG, Gerber RA, Leidy NK, Sexton CC, Karlsson J, et al. Evaluating the Power of Food Scale in obese subjects and a general sample of individuals: development and measurement properties. Int J Obes. 2009 Aug;33(8):913-22.

13 Ribeiro G, Santos O, Camacho M, Torres S, Mucha-Vieira F, Sampaio D, et al. Translation, Cultural Adaptation and Validation of the Power of Food Scale for Use by Adult Populations in Portugal. Acta Med Port. 2015 Sep-Oct;28(5):575-82.

14 Cheung LT, Ko GT, Chow FC, Kong AP. Association between hedonic hunger and glycemic control in non-obese and obese patients with type 2 diabetes. J Diabetes Investig. 2018 Sep;9(5):1135-43.

15 Yoshikawa T, Orita K, Watanabe Y, Tanaka M. Validation of the Japanese version of the power of food scale in a young adult population. Psychol Rep. 2012 Aug;111(1):253-65.

16 Fitzsimmons-Craft EE, Ciao AC, Accurso EC, Pisetsky EM, Peterson CB, Byrne CE, et al. Subjective and objective binge eating in relation to eating disorder symptomatology, depressive symptoms, and self-esteem among treatment-seeking adolescents with bulimia nervosa. Eur Eat Disord Rev. 2014 Jul;22(4):230-6.

17 Bearman SK, Stice E, Chase A. Evaluation of an intervention targeting both depressive and bulimic pathology: A randomized prevention trial. Behav Ther. 2003;34(3):277-93.

18 Guillemin F, Bombardier C, Beaton D. Cross-cultural adaptation of health-related quality of life measures: literature review and proposed guidelines. J Clin Epidemiol. 1993 Dec;46(12):1417-32.

19 Nunnally JC. Psychometric theory. 2nd ed. New York: Mc-Graw Hill; 1978.

20 Löwe B, Wahl I, Rose M, Spitzer C, Glaesmer H, Wingenfeld K, et al. A 4-item measure of depression and anxiety: validation and standardization of the Patient Health Questionnaire-4 (PHQ-4) in the general population. J Affect Disord. 2010 Apr;122(1-2):86-95.

21 Kroenke K, Spitzer RL, Williams JB, Löwe B. An ultra-brief screening scale for anxiety and depression: the PHQ-4. Psychosomatics. 2009 Nov-Dec;50(6):613-21.

22 Kliem S, Mößle T, Zenger M, Strauß B, Brähler E, Hilbert A. The eating disorder examination-questionnaire 8: A brief measure of eating disorder psychopathology (EDE-Q8). Int J Eat Disord. 2016 Jun;49(6):613-6.

23 WHO Global InfoBase team, editor. The SuRF Report 2. Surverillance of chronic disease risk factors: country level data and comparable estimates. Geneva: World Health Organization; 2005.

$24 \mathrm{Hu}$ L, Bentler PM. Cutoff criteria for fit indexes in covariance structure analysis: conventional criteria versus new alternatives. Struct Equ Modeling. 1999;6(1):1-55.

25 Clark LA, Watson D. Constructing validity: basic issues in objective scale development. Psychol Assess. 1995; 7(3):309-19.

26 Taber KS. The use of Cronbach's Alpha when developing and reporting research instruments in science education. Res Sci Educ. 2018 Dec;48(6):1273-1296.

27 MacCallum RC, Browne MW, Sugawara HM. Power analysis and determination of sample size for covariance structure modeling. Psychol Methods. 1996;1(2):130-49.

28 Schachter S. Some extraordinary facts about obese humans and rats. Am Psychol. 1971 Feb;26(2):129-44.

29 Nagl M, Hilbert A, de Zwaan M, Braehler E, Kersting A. The German Version of the Dutch Eating Behavior Questionnaire: Psychometric Properties, Measurement Invariance, and Population-Based Norms. PLoS One. 2016 Sep;11(9):e0162510.

30 Lipsky LM, Nansel TR, Haynie DL, Liu D, Eisenberg MH, Simons-Morton B. Power of Food Scale in association with weight outcomes and dieting in a nationally representative cohort of U.S. young adults. Appetite. 2016 Oct; 105:385-91.

31 Ribeiro G, Camacho M, Santos O, Pontes C, Torres S, Oliveira-Maia AJ. Association between hedonic hunger and body-mass index versus obesity status. Sci Rep. 2018 Apr;8(1):5857.

32 Vainik U, Neseliler S, Konstabel K, Fellows LK, Dagher A. Eating traits questionnaires as a continuum of a single concept. Uncontrolled eating. Appetite. 2015 Jul;90:229-39. 